\title{
Back-to-Back Correlations of Di-hadrons in dAu Collisions at RHIC
}

\author{
Anna Stasto, ${ }^{1,2,3}$ Bo-Wen Xiao, ${ }^{1,4}$ and Feng Yuan $^{5,2,4}$ \\ ${ }^{1}$ Department of Physics, Pennsylvania State University, University Park, PA 16802, USA \\ ${ }^{2}$ RIKEN BNL Research Center, Building 510A, \\ Brookhaven National Laboratory, Upton, NY 11973, USA \\ ${ }^{3}$ H. Niewodniczański Institute of Nuclear Physics, \\ Polish Academy of Sciences, Kraków, Poland \\ ${ }^{4}$ Center for High Energy Physics, Peking University, Beijing 100871, China \\ ${ }^{5}$ Nuclear Science Division, Lawrence Berkeley \\ National Laboratory, Berkeley, CA 94720, USA
}

\begin{abstract}
We perform a complete theoretical analysis of the azimuthal angular correlation of two-hadron productions in the forward $d A u$ collisions at RHIC in the saturation formalism, and obtain a very good agreement with the experimental data. It is demonstrated that the suppression and broadening of the away side peak provide a unique signal for the onset of the saturation mechanism at small- $x$ in a large nucleus. We emphasize that future experiments of di-hadron correlations in $p A$ collisions at both RHIC and LHC, and in $e A$ collisions at the planned electron-ion collider, shall provide us with a thorough study and understanding of the strong interaction dynamics in the saturation regime.
\end{abstract}


Introduction. Two particle correlations in high energy hadronic scattering processes have played important roles in studying the strong interaction QCD dynamics [1, 2], novel phenomena in proton-proton collisions [3], and the medium effects in heavy ion collisions [4-6]. In particular, the azimuthal correlation of two particles with large transverse momenta provide critical evidence of the strong jet quenching effects in the hot dense medium created in the heavy ion collisions at RHIC and the LHC. An important advantage of these measurements is that they reveal the physics by the observables themselves, which do not need, e.g., the proton reference to quantify the phenomena. It was suggested in Ref. [7] to study the cold nuclear matter in dense region by measuring the forward di-jet (di-hadron) production in proton-nucleus collisions, where the de-correlation of the back-to-back dijet in the forward $p A$ collisions can be used to signal the gluon saturation at small- $x$ in a large nucleus. This prediction was qualitatively confirmed by the STAR [8] and PHENIX [9] collaborations from di-hadron correlation measurements in $d A u$ collisions at RHIC, and have been considered as the best evidence for saturation physics. Early attempts [10, 11] have been made to understand the experimental data quantitatively, where, however, inappropriate approximations were taken in the calculations. In particular, in this paper we correctly distinguish the Weizsäcker-Williams (WW) gluon distribution from the dipole gluon distribution. In this work, we will provide, for the first time, a quantitative and thorough description of the experimental data in the saturation formalism, including the large broadening of the angular distribution and suppression of the peak for the away-side hadron. Our results emphasize the relevance of the gluon saturation in the kinematic regions covered by the STAR and PHENIX collaborations and signify the usage of the two-hadron correlation as an important tool to investigate the QCD dynamics in the small- $x$ limit.

There have been theoretical arguments [12 15] which suggest that the gluon distribution saturates at small Bjorken- $x$. The color-glass-condensate model (CGC), has been proposed to describe the gluon saturation phenomenon at small- $x$. An important feature of this approach is the appearance of the dynamically generated saturation scale $Q_{s}(x)$ which separates the dilute and dense partonic regimes. The experimental data from HERA are well described with the saturation model calculations, with the saturation scale of the order of $\sim 1 \mathrm{GeV}$. Furthermore, the forward hadron production in nucleon-nucleus $(p A)$ collisions has been systematically studied [16] in the CGC formalism, where the unintegrated gluon distributions (UGDs) are important ingredients to describe the phenomena. They unveil the 
importance of the multiple interaction effects in the factorization of the hard processes in the small- $x$ calculations. Nevertheless, there exists much more interesting dynamics [17] in saturation physics which can only be explored by di-jet or di-hadron production processes as we will demonstrate in the following calculations.

In this paper, we focus on two-particle production in the forward direction of $p A(d A u$ at RHIC) collisions,

$$
p+A \rightarrow h_{1}+h_{2}+X,
$$

where two hadrons $h_{1}$ and $h_{2}$ with large momenta are produced. The above process is sensitive to the gluon distributions at small- $x$ in the nuclear target. In order to correctly take into account the multiple interaction effects, we follow the CGC framework to calculate the two particle production [17]. An effective $k_{t}$ factorization can be established for this process in the back-to-back correlation limit, and the differential cross sections can be expressed in terms of various UGDs, which can be related to two fundamental UGDs: the dipole gluon distribution $x G^{(2)}\left(x, q_{\perp}\right)$, and the WW gluon distribution $x G^{(1)}\left(x, q_{\perp}\right)$. Only with this effective $k_{t}$ factorization, can one describe all the features (including both broadening and suppression) of the STAR [8] and PHENIX [9] data systematically. These results also agree with previous calculations for two-particle production in $p A$ collisions in the general kinematics region [18, 19].

In the RHIC experiments, the di-hadron correlations are measured by the coincidence probability $C(\Delta \phi)=N_{\text {pair }}(\Delta \phi) / N_{\text {trig, }}$, where $N_{\text {pair }}(\Delta \phi)$ is the yield of two forward $\pi^{0}$ which includes a trigger particle with a transverse momentum $p_{1 \perp}^{\text {trig }}$ and an associate particle with $p_{2 \perp}^{\text {asso }}$ and the azimuthal angle between them $\Delta \phi$. We calculate the single and two-particle cross sections and obtain,

$$
C(\Delta \phi)=\frac{\int_{\left|p_{1 \perp}\right|,\left|p_{2 \perp}\right|} \frac{d \sigma^{p A \rightarrow h_{1} h_{2}}}{d y_{1} d y_{2} d^{2} p_{1 \perp} d^{2} p_{2 \perp}}}{\int_{\left|p_{1 \perp}\right|} \frac{d \sigma^{p A \rightarrow h}}{d y_{1} d^{2} p_{1 \perp}}}
$$

where the dependence on the rapidities of the two particles is implicit.

Single inclusive cross section. Let us first discuss the single inclusive hadron production. The leading-order single inclusive cross section [16] in $p A$ collisions is given by the product of the integrated parton distributions of the projectile proton and the unintegrated gluon 
distributions of the target nucleus:

$$
\begin{aligned}
\frac{d \sigma^{p A \rightarrow h X}}{d^{2} b d^{2} p_{\perp} d y_{h}}= & \int_{z_{h}}^{1} \frac{d z_{1}}{z_{1}^{2}}\left[D_{h / q}\left(z_{1}\right) x_{p} q_{f}\left(x_{p}\right) F_{x_{g}}\left(k_{\perp}\right)\right. \\
& \left.+x_{p} g_{f}\left(x_{p}\right) \tilde{F}_{x_{g}}\left(k_{\perp}\right) D_{h / g}\left(z_{1}\right)\right]
\end{aligned}
$$

where the sum over quark flavor is implicit, $b$ represents the impact parameter in $p A$ collisions, $p_{\perp}$ and $y_{h}$ are transverse momentum and rapidity of the hadron, $q\left(x_{p}\right)$ and $g\left(x_{p}\right)$ are integrated quark and gluon distributions from the projectiles, $D(z)$ the associated fragmentation functions with $p_{\perp}=z_{1} k_{\perp}, x_{p}=p_{\perp} e^{y_{h}} / z_{1} \sqrt{s}$ and $x_{g}=p_{\perp} e^{-y_{h}} / z_{1} \sqrt{s}$. The dipole gluon distributions $F_{x_{g}}\left(k_{\perp}\right)$ and $\tilde{F}_{x_{g}}\left(k_{\perp}\right)$ are Fourier transform of the dipole scattering amplitude in the fundamental and adjoint representations, respectively. In particular, $F_{x_{g}}\left(k_{\perp}\right) \propto x_{g} G^{(2)}\left(x_{g}, k_{\perp}\right) / k_{\perp}^{2}$. In terms of the numerical study, we are able to describe the forward single hadron production cross sections measured by both BRAHMS and STAR up to $p_{\perp}=3.0 \mathrm{GeV}$ with a $\mathrm{K}$-factor about 0.8 for $y_{h}=2.0$ and 0.5 for $y_{h}=3.2$. In this numerical evaluation, we follow the NLO sets of MSTW parametrizations [20] for the parton distributions and DSS parametrizations [21] for the fragmentation functions 1 .

Two-particle production in forward $p A$ collisions. Two-particle production contains the correlated and uncorrelated contributions,

$$
d \sigma^{\left(p A \rightarrow h_{1} h_{2}\right)}=d \sigma_{\text {corr. }}^{\left(p A \rightarrow h_{1} h_{2}\right)}+d \sigma_{\text {uncorr. }}^{\left(p A \rightarrow h_{1} h_{2}\right)} .
$$

The correlated hadron production comes from the partonic $2 \rightarrow 2$ processes, where these two particles are back-to-back correlated and form the away side peak in the azimuthal angular distribution $(\Delta \phi=\pi)$. The near side correlation comes from the particle decay or the same jet fragmentation if they are at the same rapidity. In this letter, we will focus on the back-to-back correlation region, namely the away side peaks. According to Ref. [17], we can write down the differential cross section for the two-particle production in the back-to-back correlation limit,

$$
\begin{aligned}
& \frac{d \sigma_{\text {corr. }}^{\left(p A \rightarrow h_{1} h_{2}\right)}}{d y_{h_{1}} d y_{h_{2}} d^{2} p_{1 \perp} d^{2} p_{2 \perp}}=\int \frac{d z_{1}}{z_{1}^{2}} \frac{d z_{2}}{z_{2}^{2}} \frac{\alpha_{s}^{2}}{\hat{s}^{2}}\left[x_{p} q\left(x_{p}\right) \mathcal{F}_{q g}^{(i)}\right. \\
& \times H_{q g}^{(i)}\left(D_{h_{1} / q}\left(z_{1}\right) D_{h_{2} / g}\left(z_{2}\right)+D_{h_{2} / q}\left(z_{1}\right) D_{h_{1} / g}\left(z_{2}\right)\right) \\
& \left.+x_{p} g\left(x_{p}\right) \mathcal{F}_{g g}^{(i)} H_{g g}^{(i)} D_{h_{1} / g}\left(z_{1}\right) D_{h_{2} / g}\left(z_{2}\right)\right],
\end{aligned}
$$

\footnotetext{
1 A recent next-to-leading order calculation for inclusive hadron production suggests that the appropriate choice for the factorization scale to be around the saturation scale [22]. We have followed this choice in our calculations.
} 
where $x_{g}=x_{1} e^{-y_{1}}+x_{2} e^{-y_{2}}$ and $x_{p}=x_{1} e^{y_{1}}+x_{2} e^{y_{2}}$ with $x_{i}=\left|k_{i \perp}\right| / \sqrt{\hat{s}}$ and $k_{i \perp}=p_{i \perp} / z_{i}$, $\mathcal{F}^{(i)}$ and $H^{(i)}$ are various UGDs and the associated hard coefficients, respectively. Their expressions can be found in Ref. [17]. The partonic center of mass energy squared $\hat{s}$ is defined as $\hat{s}=P_{\perp}^{2} / z(1-z)$ with $P_{\perp}=\left(k_{1 \perp}-k_{2 \perp}\right) / 2$ and $z=x_{1} e^{y_{1}} / x_{2} e^{y_{2}}$. In the CGC calculations [17], $\tilde{P}_{\perp}=(1-z) k_{1 \perp}-z k_{2 \perp}$ also enters in the hard coefficients, which equals to $P_{\perp}$ in the correlation limit. The difference between $P_{\perp}$ and $\tilde{P}_{\perp}$ will be used to estimate the theoretical uncertainties in the following calculations. In the typical kinematics of the forward collisions at RHIC, we find that $x_{p} \sim 0.1$ and $x_{g} \leq 10^{-3}$, where both the quark initiated processes $(q \rightarrow q g$ channel) and gluon initiated processes $(g \rightarrow g g)$ contribute.

Comparing the above equation to Eq. (3) of Ref. [10], one immediately finds notable differences between the results. In particular in Ref. [10] the only channel calculated was $q \rightarrow q g$. Moreover, in this channel our results do not agree with results in Ref. [10] since in the latter work the contributions from the WW gluon distribution were not taken into account. These contributions are essential in order to reproduce correctly the collinear factorization results for dijet production in the dilute limit.

The unintegrated gluon distributions in Eq. (5) are largely un-explored, in particular, for those related to the WW gluon distribution. The energy evolution is important to understand their behavior depending on $x_{g}$, of which for the dipole gluon distribution, the Balitsky-Kovchegov (BK) evolution, has been well studied [15] and demonstrated the socalled geometric scaling [23] in the solution. The scaling was found to be related to the traveling wave solutions [24, 25] of the BK evolution. The energy evolution equation for the WW gluon distribution has recently been systematically investigated [26]. An important result from these studies is the geometric scaling similar to the dipole gluon distribution. Therefore, as a first step, we can parametrize these gluon distributions from a model calculation, and include the energy dependence by assuming the geometric scaling and $x_{g}$-dependence of the saturation scale. In the following, we adopt the Golec-Biernat Wusthoff model [27] for the dipole gluon distribution which successfully describes the low- $x$ DIS structure functions at HERA, then extend it to the WW gluon distribution and include the nuclear dependence by modifying the saturation scale as [15]

$$
Q_{s A}^{2}=c(b) A^{1 / 3} Q_{s}^{2}(x)
$$

where $c(b)$ represents the profile function of nucleus depending on the impact parameter $b$ of 
the collision, $Q_{s}^{2}(x)=Q_{s 0}^{2}\left(x / x_{0}\right)^{-\lambda}$ with $Q_{s 0}=1 \mathrm{GeV}, x_{0}=3.04 \times 10^{-3}$ and $\lambda=0.288$ follow GBW parameterizations [27]. The profile function $c(b)$ is closely related to the centrality of the $p A$ (or $d A$ ) collisions. Central collisions give large value of $c(b)$, while peripheral collisions correspond to small profile function.

We would like to emphasize that the GBW model is not sufficient to describe the UGDs in the region that $k_{\perp}$ is much larger than $Q_{s}$. However, for the forward $p A$ collisions, the saturation scale $Q_{s}$ is large enough to cover most of the kinematics where $k_{\perp}$ is around $Q_{s}$ and we will be able to well describe the experimental data. For $p p$ collisions, we have to either modify the GBW model or include the broadening effect from the fragmentation function to describe the experimental data.

Double parton scattering contribution. Now, we turn to the un-correlated two-particle production in the process (11). This part mainly comes from two independent hard scatterings, which is referred as double parton scattering (DPS) contributions (see recent developments [28-32]). It has been pointed out in Ref. [28] that the DPS may exceed the single parton scattering contribution in the forward $p A$ collisions. Following these ideas, we estimate its contributions in $p A$ collisions in the saturation formalism. In particular, the multiple interactions from the nuclei side has been taken into account in the CGC factorization formalism [15]. For the proton side, we follow a simple parametrization for the double parton distribution: $\mathcal{D}_{p}^{i j}\left(x_{p}, x_{p}^{\prime}\right)=\mathcal{C}\left(x_{p}, x_{p}^{\prime}\right) f_{i}\left(x_{p}\right) \times f_{j}\left(x_{p}^{\prime}\right)$ with $\mathcal{C} \approx 1$, where $i$ and $j$ represent the two partons from the nucleon which participate the hard scattering, $x_{p}$ and $x_{p}^{\prime}$ for their momentum fractions. The final expression reads as

$$
\begin{gathered}
\frac{d \sigma_{\text {uncorr. }}^{\left(p A \rightarrow h_{2} h_{2}\right)}}{d^{2} b d y_{h_{1}} d y_{h_{2}} d^{2} p_{1 \perp} d^{2} p_{2 \perp}}=\int \frac{d z_{1}}{z_{1}^{2}} \frac{d z_{2}}{z_{2}^{2}} D\left(z_{1}\right) D\left(z_{2}\right) \\
\times \sum_{i j} x_{p} f_{i}\left(x_{p}\right) x_{p}^{\prime} f_{j}\left(x_{p}^{\prime}\right) F_{x_{g}}^{(i)}\left(k_{1 \perp}\right) F_{x_{g}^{\prime}}^{(j)}\left(k_{2 \perp}\right),
\end{gathered}
$$

where $i j$ represent flavors of the partons from the nucleon and the associated UGDs from nuclei, and $x_{p}$ and $x_{g}$ are determined by the kinematics of the two hard scatterings.

An important feature of the above DPS contribution is that the two hard scatterings are independent to each other at the leading order approximation [28 -30]. Therefore, the two particles in the final state are un-correlated, and their azimuthal angle distribution will be flat. This leads to the so-called pedestal contribution in the experimental measurements.

Comparison with the experimental data. With the above formulas, we are ready to compare to the experimental data on the two-particle correlation measurements in the forward 

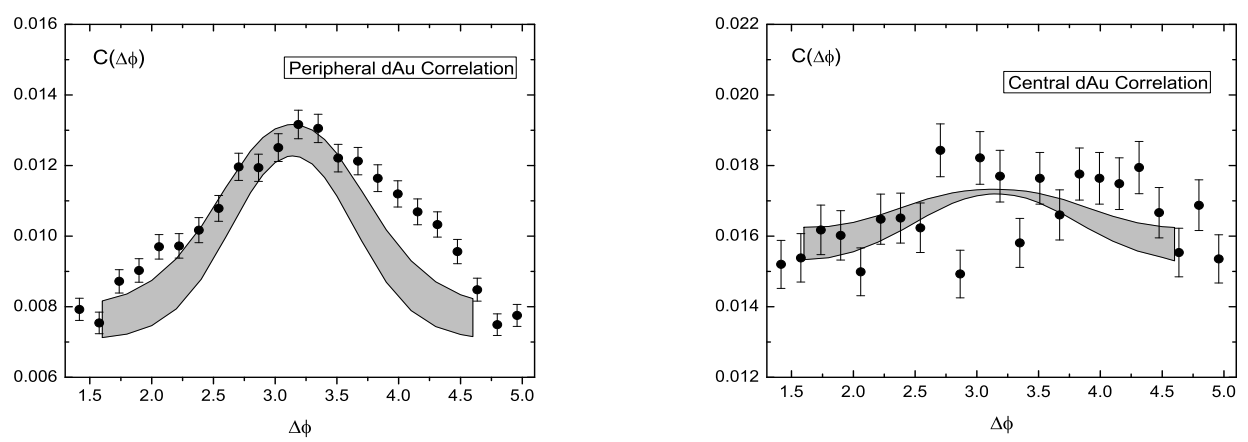

FIG. 1. The forward di-pion correlations $C(\Delta \phi)$ of Eq. (2) at $y_{1} \sim y_{2} \sim 3.2$ in peripheral and central $d A u$ collisions compared to the preliminary data from the STAR collaboration [8]. Centrality definition follows Ref. [8], where the average impact parameters are found around $6.7 \mathrm{fm}$ and $2.7 \mathrm{fm}$ accordingly. The grey error band comes from using $P_{\perp}$ or $\tilde{P}_{\perp}$ in the hard coefficients in Eq. (5).

$d A u$ collisions at RHIC. Before we do that, we would like to emphasize that the saturation scale plays a key role in describing the correlation $C(\Delta \phi)$ of the away side peak, including both broadening and the suppression. First, the width of the away side peak will increase with the saturation scale because of the broadening effects. Quantitatively, the effective $k_{t}$-factorization formula of Eq. (5) lead to stronger broadening effects compared to the naive $k_{t}$-factorization calculations. This is because the various gluon distributions contain the convolution of the UGDs and will enhance the broadening. Without this enhancement, we can not describe the broadening effects. In particular, when the saturation scale reaches the transverse momenta of the dijet, the away side peak will almost disappear as indicated in the experimental data for the central collisions at RHIC and the theory calculations as well.

Second, the magnitude of the correlation $C(\Delta \phi)$ is also sensitive to the saturation scale $Q_{s}$. In particular, larger $Q_{s}$ push the dipole gluon distribution to larger transverse momentum, which leads to single particle production (3) increasing with $Q_{s}$. The correlated two-particle production cross section (5), however, decreases with $Q_{s}$ for the same reason. Therefore, the correlated contribution to $C(\Delta \phi)$ decreases accordingly. Our numeric evaluation also supports this conclusion. On the other hand, the un-correlated two particle production cross section (7) roughly depends on the product of two single particle cross sections. Therefore, its contribution increases more rapidly with $Q_{s}$ than that of single particle cross section. The consequence is that the pedestal contribution increases with $Q_{s}$. 
All these features are evident when we compare to the STAR data [8]. As an example, we show in Fig. 1 the results for $p_{1 \perp}^{\text {trig }}>2 \mathrm{GeV}$ and $1 \mathrm{GeV}<p_{2 \perp}^{\text {asso }}<p_{1 \perp}^{\text {trig }}$ at $y_{1} \sim y_{2} \sim 3.2$ in the peripheral and central collisions, respectively. In our calculations, we assume a fixed strong coupling constant $\alpha_{s}=0.35$. The saturation scale $Q_{s}(c(b)$ in Eq. ([6]) ) is the only parameter to fit the data, for which we found $c(b)=0.45,0.56$, 0.85 for the peripheral, minimum bias, and central collisions, respectively. These parameters are consistent with $c(0) \approx 0.9$ and the centrality dependence of the nuclei profile for these collisions, by using either the hard sphere model $c(b)=c(0) \sqrt{1-b^{2} / R_{A}^{2}}$ or the Wood-Saxon model. The pedestal contributions to $C(\Delta \phi)$ are found to be around 0.016 and 0.018 for the peripheral and central collisions, which are in rough agreement with the experimental measurements. In the plots of Fig. 1, in order to better compare the results, we used the experimental extractions of pedestal contributions. The grey error band of the theory calculations comes from the difference between $P_{\perp}$ and $\tilde{P}_{\perp}$ used in the hard coefficients in Eq. (5). Similar results are also found when we compare to the correlation measurements from PHENIX collaboration.

It is important to note that in the central $d A u$ collisions the disappearing of the away-side peak in the di-hadron production indicates that the saturation scale is the same order as the hard probe of the jet transverse momentum, which is a clear signal of the onset of the saturation mechanism for this observable. From the kinematics of this collision, we conclude that the saturation scale $Q_{s}$ reaches at $\sim 2 \mathrm{GeV}$ at $x_{g} \sim 6 \times 10^{-4}$ in the center of the gold nucleus with jet transverse momentum $k_{\perp} \sim 3 \mathrm{GeV}$ at rapidity 3.2. We hope that the future measurements at RHIC and LHC will provide more information and help to map out the complete phase structure of the cold nuclei at small- $x$. We want also to emphasize that the above conclusion is very general and independent of the model we used for the UGDs. As mentioned above, the GBW model captures the main features for the UGDs at the transverse momentum around the saturation scale in which most of the data exist.

Meanwhile, the PHENIX collaboration has also reported the nuclear suppression factor $J_{d A}$ defined as

$$
J_{d A}=\frac{1}{\left\langle N_{\text {coll }}\right\rangle} \frac{\sigma_{d A}^{\text {pair }} / \sigma_{d A}}{\sigma_{p p}^{\text {pair }} / \sigma_{p p}},
$$

where $\sigma^{\text {pair }}$ is the cross section of two-particle production in $d A$ and $p p$ collisions with the pedestal contributions subtracted. $\sigma_{d A}$ and $\sigma_{p p}$ are the cross sections for the full event selection. In the absence of gluon saturation and nuclear effects, the dihadron cross-sections 


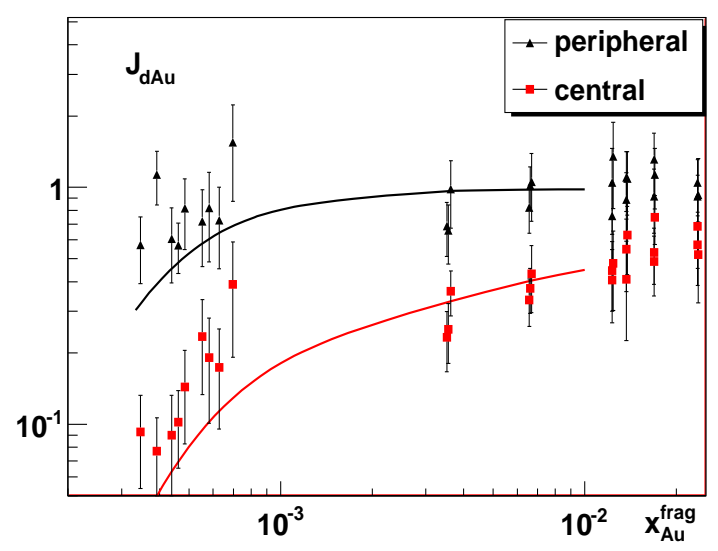

FIG. 2. The nuclear suppression factor $J_{d A}$ of Eq. (8) for the two particle production in $d A u$ collisions as function of $x_{\mathrm{Au}}^{\mathrm{frag}}$. The experimental data from PHENIX [9] and theory calculations are pedestal contributions subtracted.

are expected to scale with $\left\langle N_{\text {coll }}\right\rangle$. Therefore, $J_{d A}$ should be equal to unity in the dilute regime and suppressed in the dense regime. The experimental kinematic variable $x_{\mathrm{Au}}^{\mathrm{frag}}=$ $\left(p_{1 \perp} e^{-y_{1}}+p_{2 \perp} e^{-y_{2}}\right) / \sqrt{s}$ has been used to represent the $x$-dependence [9]. In Fig. 2, we calculated $J_{d A}$ as function of $x_{\mathrm{Au}}^{\text {frag }}$ for a typical transverse momentum $p_{1 \perp}=p_{2 \perp}=1.0 \mathrm{GeV}$ with the same rapidity for the two particles, for central collision $(c(b)=0.85)$ and peripheral collision $(c(b)=0.45)$, where we have used $\tilde{P}_{\perp}$ in the hard coefficients in Eq. (5). Similar results are obtained with choice of $P_{\perp}$. As a comparison, we list the PHENIX data in Fig. 2, where the data are for different values of $p_{i \perp}$ and rapidities. From this figure, we clearly see that the the suppression of $J_{d A}$ at low $x_{\mathrm{Au}}^{\mathrm{frag}}$ due to the saturation of the cross sections in $p p$ and $d A$ collisions, as also indicated by the experimental data. However, we emphasize that $J_{d A}$ depends on the $p p$ reference, for which our model calculations should be taken with cautions.

In summary, we have carried out a complete numerical study on the forward dihadron correlations in $d A u$ collisions and found good agreement with the experimental data from RHIC. These results demonstrated that the saturation formalism developed recently can be used to describe the broadening and suppression of the away side peak in the di-hadron production in $p A$ collisions. This emphasizes that the di-hadron (dijet) correlation provides a unique signal for the onset of saturation mechanism at small- $x$ in a large nucleus. Future 
experiments at RHIC, EIC and LHC will provide excellent opportunities to thoroughly investigate the QCD dynamics in the saturation regime.

We thank E. Avsar, L. Bland, M. Chiu, F. Dominguez, C. Marquet, L. McLerran, A. H. Mueller, J.W. Qiu, M. Strikman, R. Venugopalan, W. Vogelsang and N. Xu for helpful conversations. In particular, we are grateful to R. Venugopalan for stimulating discussions and comments. This work was supported in part by the U.S. Department of Energy under the contracts DE-AC02-05CH11231 and DOE OJI grant No. DE - SC0002145. We are grateful to RIKEN, Brookhaven National Laboratory and the U.S. Department of Energy (contract number DE-AC02-98CH10886) for providing the facilities essential for the completion of this work.

[1] V. M. Abazov et al. [ D0 Collaboration ], Phys. Rev. Lett. 94, 221801 (2005).

[2] V. Khachatryan et al. [ CMS Collaboration ], Phys. Rev. Lett. 106, 122003 (2011).

[3] V. Khachatryan et al. [ CMS Collaboration ], JHEP 1009, 091 (2010).

[4] J. Adams et al. [ STAR Collaboration ], Phys. Rev. Lett. 91, 072304 (2003).

[5] G. Aad et al. [ Atlas Collaboration ], Phys. Rev. Lett. 105, 252303 (2010).

[6] S. Chatrchyan et al. [ CMS Collaboration ], Phys. Rev. C84, 024906 (2011).

[7] C. Marquet, Nucl. Phys. A 796, 41 (2007).

[8] E. Braidot, for the STAR Collaboration, Nucl. Phys. A 854, 168 (2011).

[9] A. Adare et al. [PHENIX Collaboration], Phys. Rev. Lett. 107, 172301 (2011).

[10] J. L. Albacete and C. Marquet, Phys. Rev. Lett. 105, 162301 (2010).

[11] K. Tuchin, Nucl. Phys. A846, 83-94 (2010).

[12] L. V. Gribov, E. M. Levin and M. G. Ryskin, Phys. Rept. 100, 1 (1983).

[13] A. H. Mueller and J. w. Qiu, Nucl. Phys. B 268, 427 (1986).

[14] L. D. McLerran and R. Venugopalan, Phys. Rev. D 49, 2233 (1994); Phys. Rev. D 49, 3352 (1994).

[15] F. Gelis, et al., Ann. Rev. Nucl. Part. Sci. 60, 463-489 (2010); and references therein.

[16] A. Dumitru and J. Jalilian-Marian, Phys. Rev. Lett. 89, 022301 (2002); J. L. Albacete, C. Marquet, Phys. Lett. B687, 174-179 (2010).

[17] F. Dominguez, B. W. Xiao and F. Yuan, Phys. Rev. Lett. 106, 022301 (2011); F. Dominguez, 
et al., Phys. Rev. D 83, 105005 (2011).

[18] J. P. Blaizot, F. Gelis and R. Venugopalan, Nucl. Phys. A 743, 57 (2004).

[19] J. Jalilian-Marian and Y. V. Kovchegov, Phys. Rev. D 70, 114017 (2004) [Erratum-ibid. D 71, 079901 (2005)].

[20] A. D. Martin, W. J. Stirling, R. S. Thorne and G. Watt, Eur. Phys. J. C 63, 189 (2009).

[21] D. de Florian, R. Sassot and M. Stratmann, Phys. Rev. D 75, 114010 (2007); Phys. Rev. D 76, 074033 (2007).

[22] G. A. Chirilli, B. -W. Xiao and F. Yuan, arXiv:1112.1061 [hep-ph].

[23] A. M. Stasto, K. J. Golec-Biernat and J. Kwiecinski, Phys. Rev. Lett. 86, 596 (2001).

[24] S. Munier and R. B. Peschanski, Phys. Rev. Lett. 91, 232001 (2003); Phys. Rev. D 69, 034008 (2004).

[25] A. H. Mueller, D. N. Triantafyllopoulos, Nucl. Phys. B640, 331-350 (2002). hep-ph/0205167.

[26] F. Dominguez, et al., Phys. Lett. B 705, 106 (2011); A. Dumitru, et al., Phys. Lett. B 706, 219 (2011); E. Iancu and D. N. Triantafyllopoulos, JHEP 1111, 105 (2011).

[27] K. J. Golec-Biernat and M. Wusthoff, Phys. Rev. D 59, 014017 (1998).

[28] M. Strikman and W. Vogelsang, Phys. Rev. D 83, 034029 (2011).

[29] J. R. Gaunt, et al., Eur. Phys. J. C 69, 53 (2010); and references therein.

[30] M. Diehl and A. Schafer, Phys. Lett. B 698, 389 (2011).

[31] J. R. Gaunt, W. J. Stirling, JHEP 1003, 005 (2010).

[32] B. Blok, Y. Dokshitzer, L. Frankfurt, M. Strikman, Phys. Rev. D83, 071501 (2011). 